\title{
Pathway-pathway network-based study of the therapeutic mechanisms by which salvianolic acid $B$ regulates cardiovascular diseases
}

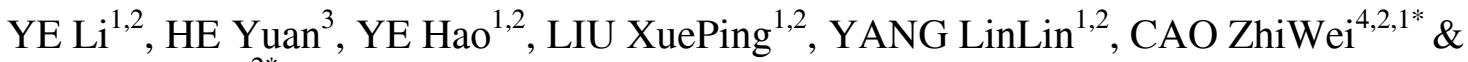 \\ TANG KaiLin ${ }^{2 *}$ \\ ${ }^{1}$ State Key Laboratory of Bioreactor Engineering, East China University of Science \& Technology, Shanghai 200237, China; \\ ${ }^{2}$ Shanghai Center for Bioinformation Technology, Shanghai 200235, China; \\ ${ }^{3}$ Department of Periodontology \& Oral Medicine, School of Stomatology, Tongji University, Shanghai 200092, China; \\ ${ }^{4}$ School of Life Science and Technology, Tongji University, Shanghai 200092, China
}

Received October 28, 2011; accepted December 12, 2011

\begin{abstract}
Investigation of the potential therapeutic mechanisms of drug candidates is an essential step in the process of new drug discovery. With the rapid development of systems biology, recent network analyses of proteins, drugs, and diseases have enabled great progress in delineating the molecule mechanisms of drug candidates. However, most analyses perform a direct association between gene/protein and disease levels without considering the intermediate biological pathways regulated by the drugs. Given that a protein performs its biological roles through pathways, we propose using a novel pathway-pathway network analysis to investigate the potential therapeutic functions of the drug candidates. Many studies have demonstrated that salvianolic acid B (Sal B) of Salvia miltiorrhiza is an effective therapy for cardiovascular diseases (CVD). Using molecular docking methods to identify direct interacting targets of Sal B, we collected all Sal B-regulated proteins with supporting experimental evidence in PubMed abstracts. FDA-approved CVD drugs and their corresponding targets were also collected. From a traditional drug-protein network analysis, we found that Sal B could affect ACE and REN of the renin-angiotensin-aldosterone system to relax vessels and alleviate hypertension. Subsequent pathway-pathway network analysis was attempted to study the mechanisms of Sal B in treating CVD, and demonstrated that Sal B regulates immunity/inflammation, apoptosis, ion transport and basic metabolism processes in the treatment of CVD. Regulating the immune/inflammation process may be the major mechanism of Sal B. We believe that pathwaypathway network analysis is a novel method for studying the therapeutic mechanisms of herbal ingredients.
\end{abstract}

cardiovascular diseases, salvianolic acid B, network analysis, biological pathway network, module analysis

Citation: Ye L, He Y, Ye H, et al. Pathway-pathway network-based study of the therapeutic mechanisms by which salvianolic acid B regulates cardiovascular diseases. Chin Sci Bull, 2012, 57: 1672-1679, doi: 10.1007/s11434-012-5142-y

Defining the mechanism by which candidate drugs act is an indispensable step in the process of drug discovery. Traditional discovery methods often produce relatively accurate outcomes but usually require huge expenditure of time and money. In recent years, network analyses based on protein-protein interactions, drug-drug association networks and disease-drug association networks have achieved good

*Corresponding authors (email: zwcao@tongji.edu.cn; kltang@scbit.org) results in researching the mechanisms by which candidate drugs exert therapeutic effects in disease [1-5]. Network analyses based on protein-protein interactions mainly study topological characteristics of targets or proteins influenced by drugs in a given biological network, or the relative distance between disease-associated proteins, to deepen the understanding of the molecular mechanisms of drugs [1-3]. For example, Yue et al. [6] constructed a network consisting of all possible ganoderic acid D (GAD) targets, all of which 
were supported by experimental evidence, then analyzed the relationships between these proteins and the cytotoxicity of GAD on the network. Other methods help us to understand the therapeutic mechanism of drugs by analyzing drug-drug association networks, which are constructed with drugs targeting the same disease. This method predicts that drugs with the same therapeutic effect may also share a common therapeutic mechanism by targeting not only known drug targets, but also by interacting unexpectedly with a shared "cryptic" target(s) [4]. In addition, analyzing the associated network of genes/proteins, drugs or diseases based on the similarity of gene-expression profile signatures can predict possible influences of known drugs on other diseases, and facilitate a full understanding of the regulatory mechanisms employed by these drugs [5].

Although significant developments have been made in studying the therapeutic mechanisms of drugs using analytical methods to interrogate biological association networks, most analyses perform a direct association from the gene/ protein level to the disease level. However, there is a considerable gap between single proteins and physiological or disease symptoms, which limits the degree to which these predictions can be translated to the whole organism. In vivo, a biologically active compound usually associates with many genes or proteins, each of which can be related to a variety of diseases, which can confuse efforts to define the predicted pharmacology of a bioactive compound. Proteins usually perform their physiological function by forming protein complexes or participating in biological signaling pathways [7]. Disease is often a much more complex physiological phenomenon, its pathological processes generally being related to many abnormal biological pathways. To overcome this deficiency, pathway-pathway associated networks have been proposed that are composed of many biological pathways based on mutual links or crosstalk, and that can better reflect the complexity up- and down-stream of a given target within a network of human biological pathways. As biological pathway research advances, an increasing number of reliable biological pathways with credible information regarding mutual crosstalk have been identified, which should greatly facilitate the construction of biological pathway-associated networks [8].

In general, Western drugs are designed to be monotherapies (i.e. single active ingredient acting at a single target). In contrast, traditional Chinese medicine incorporates a variety of ingredients that can synergistically regulate various related biological pathways through multiple targets. This synergy may contribute to the ultimate therapeutic effect [9-11]. Therefore, applying biological pathway-associated network analyses to the study of the molecular mechanisms of drug action seems suited to traditional Chinese medicines.

Many pharmacological studies have discovered that salvianolic acid B (Sal B), a principal component of Salvia miltiorrhiza, is efficacious in curing cardiovascular disease
(CVD) [12,13], a conclusion supported by US FDA phase II clinical trials. In addition, some researchers suggested that many cellular functions such as signal transmission are achieved by the mutual interaction of many biological modules, each with their own specific physiological function [14]. In this study, we used Sal B as an example to investigate the relationship between functional modules and CVD based on a pathway-pathway association network, constructed using methods of target prediction, network assembly and decomposition.

\section{Methods}

\subsection{In silico salvianolic acid B target screening and collection of other regulated proteins}

In the field of molecular modeling, docking methods are used to predict the preferred orientation of one molecule in relation to a second when bound to each other in a stable complex. Molecular docking is used to evaluate the binding affinity of ligands and proteins according to space and energy matching, and is widely applied in molecular screening. Several excellent software programs have been developed, such as DOCK [15], AutoDock [16] and FleX [17]. INVDOCK [18] is the first reverse-docking software, which has been successfully used in potential target screening. Yue et al. [6] investigated the cellular toxicity of GAD based on 21 potential targets identified by INVDOCK. In the current study, we collected all proteins with elaborate PDB structure which participated in 17 CVD-related GO [19] processes (see Table 1, http://wiki.geneontology.org/index. php/Defining_CV-associated_gene_list). INVDOCK screening was then used to identify potential targets of Sal B. Additionally, Sal B-regulated proteins were manually curated from the literature in Medline. To investigate the veracity of the relationship between the predicted targets and CVD, the distance between predicted targets and regulated proteins in the PPI (protein-protein interaction) network. The interacted protein-protein pairs were extracted from KEGG (Kyoto Encyclopedia of Genes and Genomes).

\subsection{Sal B target-drug association network}

The Anatomical Therapeutic Chemical (ATC) classification system is used for the classification of drugs. The drugs are divided into 14 main groups (1st level). The first level of the code indicates the anatomical main group and consists of one letter, for example, the ATC-code started with letter ' $C$ ' indicates that the agent is used for cardiovascular system disease. The curated CVD drugs whose first letter of ATC code is " $\mathrm{C}$ " and their corresponding targets (as verified by the FDA) were collected from DrugBank. The Sal B targetdrug association network was then built based on the overlap targets of Sal B and CVD drugs. The Sal B target-drug association network was then built based on the overlap 
Table 1 CVD related GO items

\begin{tabular}{cl||ll}
\hline \multicolumn{1}{c||}{ GO ID } & \multicolumn{1}{c}{ GO term } & \multicolumn{1}{c}{ GO ID } & \multicolumn{1}{c}{ GO term } \\
\hline GO:0007507 & Heart development & GO:0006979 & Response to oxidative stress \\
GO:0048738 & Cardiac muscle development & GO:0006629 & Coagulation \\
GO:0008015 & Circulation & GO:0006936 & Mipid metabolism \\
GO:0050878 & Regulation of body fluids & GO:0048771 & Tissue remodeling \\
GO:0001944 & Vasculature development & GO:0051145 & Smooth muscle cell differentiation \\
GO:0042060 & Wound healing & GO:0007517 & Muscle development \\
GO:0006979 & Response to oxidative stress & GO:0042692 & Muscle cell differentiation \\
GO:0016055 & Wnt receptor signaling pathway & & \\
GO:0048659 & Smooth muscle cell proliferation & & \\
\hline
\end{tabular}

targets of Sal B and CVD drugs.

\subsection{Pathway-pathway association network for Sal B}

To investigate the mechanism of Sal B in exerting a biological effect at the pathway level, we removed the pathological disease pathways in KEGG, including several basic pathways. A gene set enrichment analysis based on a hyper-geometric test was implemented toward the basic pathways. The pathway-pathway association network was constructed by creating targeted pathway interaction pairs for Sal B, according to the pathway-pathway interactions defined in KEGG. In the network, each node represents a pathway, while each edge denotes a pathway-pathway interaction. We then applied a simulated annealing algorithm to break up this network according to the minimization of modularity metric [20,21]. In addition to the regulated proteins, the category information of pathways is considered in finding the primary biological process in each module.

\section{Results}

\subsection{Statistical information on predicted and proven targets of Sal B}

In the Gene Ontology (GO) database, we gathered 1985 CVD-associated human proteins based on $17 \mathrm{GO}$ biological process terms related to CVD development. Fifty-eight Sal $\mathrm{B}$ targets were found by INVDOCK within 835 proteins whose $3 \mathrm{D}$ structural resolution was no more than $3 \AA$. According to literature curated in PubMed, 11 experimentally proven protein targets regulated by Sal B in the treatment of CVD were collected and only a single regulated protein matched the predicted target set.

It is well known that the targets predicted by docking are all direct targets. That is to say, only the proteins which physically bind with small molecule are identified as the potential targets. On the other hand, the regulated proteins curated from literatures only indicated that Sal B could influence the activity of the protein, whether their physically binding with Sal B is not required. The regulated proteins are not only the direct targets but also indirect targets or secondary targets. The regulated proteins may be close to the predicted targets. Therefore, we calculated the distances between the predicted targets and the regulated proteins in protein-protein interaction network. Among the 11 regulated proteins, 5 are 1 step from the predicted targets; 3 are 2 steps from the predicted targets; 1 is 3 steps from the predicted targets; the last 1 protein is not in the network. About $82 \%$ regulated proteins are located within two steps from their predicted targets. They might be the secondary targets affected by the direct targets. This may imply that Sal B might regulate CVD through these 58 targets.

\subsection{Analysis of target-drug association network}

In the DrugBank database, we found 160 FDA-approved small molecule drugs that are currently used to treat CVD and 193 corresponding targets. A Sal B drug-target association network (Figure 1) was constructed based on the common targets between Sal B and the 160 drugs. In Figure 1, 9 common targets and 23 CVD drugs are covered, with 2 common targets overlapped between Sal B and Moexipril. Moexipril is considered to be an inhibitor of angiotensin-converting enzyme (ACE), which may regulate CVD similarly to hypertension or stroke by relaxing blood vessels to lower blood pressure. As a part of the renin-angiotensinaldosterone system (RAAS), the ACE enzyme is responsible for the translation of angiotensin I to angiotensin II and inactivation of bradykinin [22]. ACE inhibitors that decrease the amount of angiotensin II and elevate bradykinin are often used to treat hypertension and coronary artery diseases. Sal B is related to moexipril through the vasoconstriction effects associated with ACE, and ACE2 is classed as both a successful target (hit by an FDA-approved drug) and a research target (hit by a research drug). Given this status, it could be concluded that Sal B acts via a similar therapeutic mechanism to moexipril (i.e. anti-vasoconstriction through the inhibition of ACE) in treating CVD. Indeed, it has been shown that the aqueous extract of Salvia 


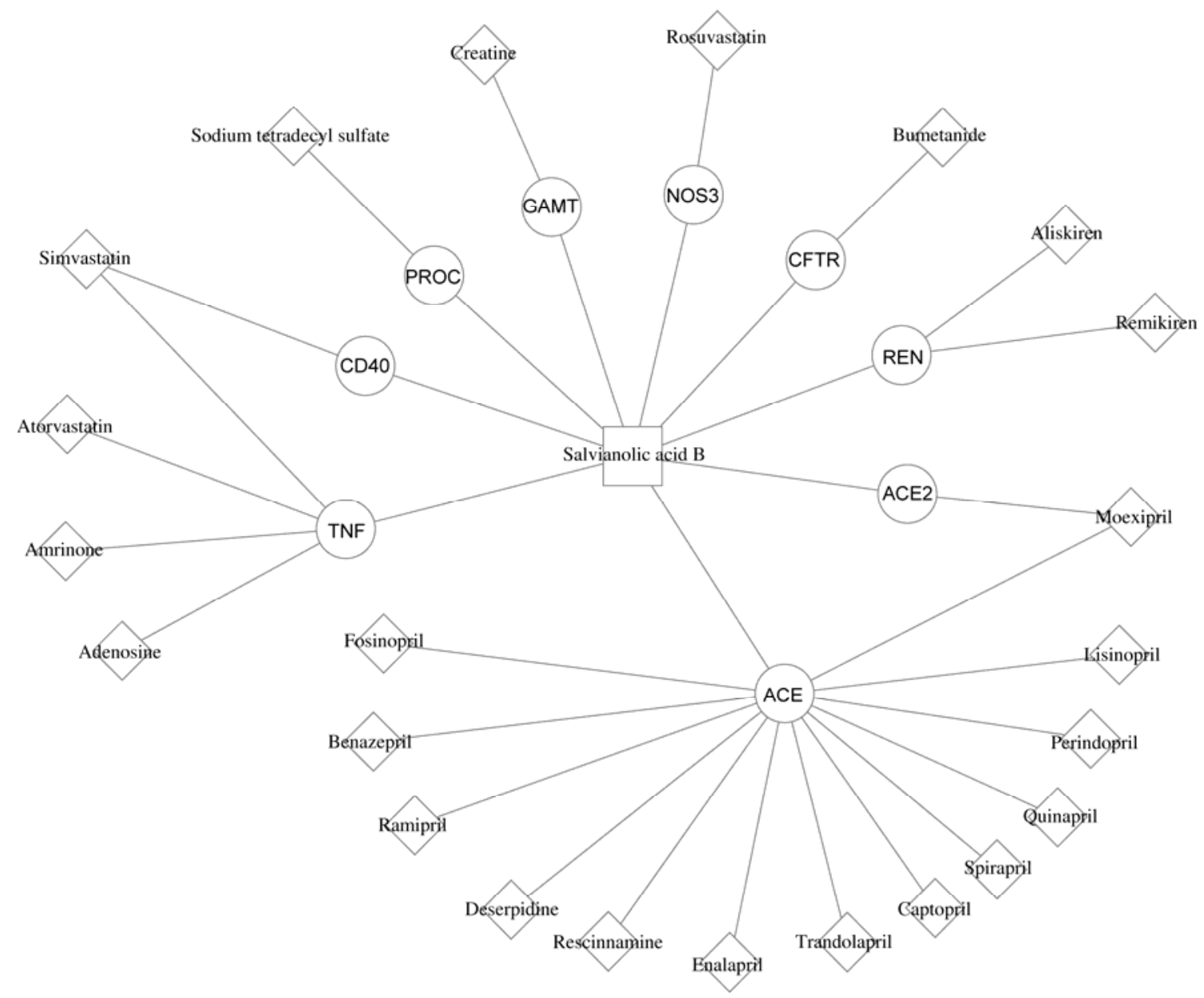

Figure 1 The Sal B-target-drug association network. The square represents Sal B; circles are common targets between Sal B and drugs; diamonds are CVD therapeutic drugs. This figure was produced using Cytoscape software [24].

miltiorrhiza, which includes Sal B, can inhibit ACE activity in rabbit lung tissue [23]. In addition, renin (REN), an important component of RAAS, is involved in the translation of angiotensinogen to angiotensin I and the development of vasoconstriction. The drugs remikiren and aliskiren exert anti-hypertensive effects by regulating REN. Given the relationship of Sal B to these drugs, and its effect on REN, it could be suggested that Sal B regulates hypertension in a similar way, by interfering with REN.

Figure 1 also shows several statin drugs such as simvastatin, rosuvastatin and atorvastatin that treat CVD primarily by inhibiting the activation of HMG-CoA reductase. Considering that the common targets (TNF, CD40, NOS3) shared by Sal B and these drugs are not directly associated with the metabolism of cholesterol, it is unlikely that Sal B generates the same biological function as these statins.

\subsection{Analysis of pathway-pathway association network of Sal B}

The above analysis revealed potential parts of the biological function of Sal B, but this small number of common targets cannot provide comprehensive information for us to define comprehensively its therapeutic mechanism. Therefore, the pathway-pathway association network method was adopted to study the molecular mechanism of Sal B. First, mapping 58 targets into the KEGG Pathway database, we retrieved 86 basic pathways. The pathway-pathway association network was constructed based on information regarding the connectivity of these 86 pathways, and the resultant network contained 86 nodes and 190 edges. Eighty-two nodes are included in the largest component sub-network. There are also four isolated pathways in the network (Drug metabolism—other enzymes; ABC transporters; Hematopoietic cell lineage; and Dorsoventral axis formation). Detailed information is listed in Figure S1.

With the aim of minimizing the modularity metric, the simulated annealing algorithm was used to decompose the network. As shown in Figure 2, the network breaks down into six modules with a modular coefficient of 0.497 and $Z$ score of 12.315 , compared with an average modular coefficient of 0.008 for 10000 random cases with the same number of nodes and degree of distribution. According to the pathway classification information in the KEGG Pathway 


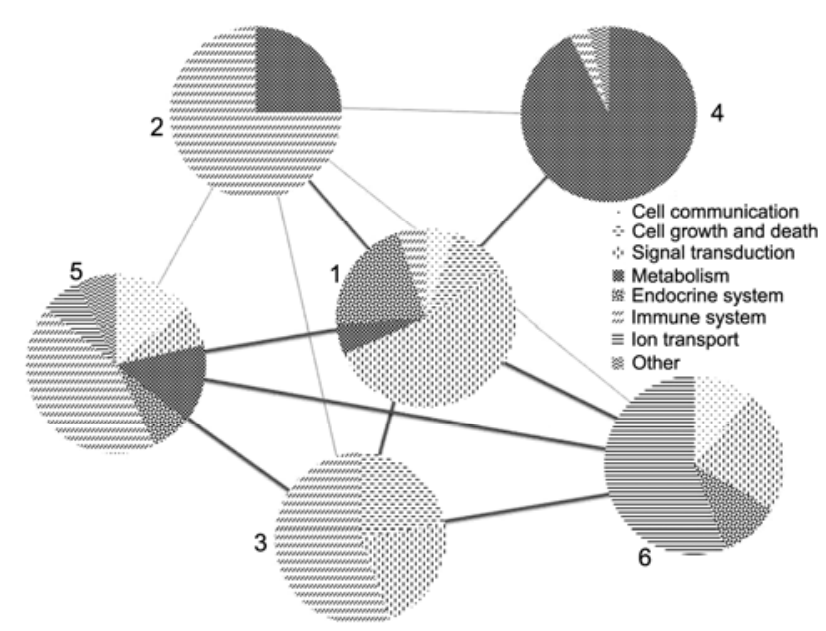

Figure 2 Modularization of the pathway-pathway association network regulated by Sal B. Each circle represents a module in the pathway-pathway association network. The subdivision of each module reflects the relative representation of basic pathways within each of seven specified biological processes and an "Other" group. Edges are suggestive of a linkage between two modules and the thickness of edge reflects the number of linkages between them.

database, 7 representative biological processes were assigned to the 82 basic pathways. These were: cell communication; cell growth and death; signal transduction; metabolism system; endocrine system; immune system; and ion transport system. Pathways not assigned into these 7 classes were denoted as "Other". Detailed information is listed in Table S1.

In Figure 2, it was clear that modules 1, 2, 3, 5 and 6 had relatively high network connectivity; module 1 was the most closely connected. In the modularized network, each module was composed of various basic pathways belonging to different biological processes, the ratio of which varied between modules. Modules 1 and 5 contained a larger number of biological processes than other modules, which implied that the two modules carried out relatively more complex biological functions. In module 1, 19 pathways were classed into 6 biological processes, with 10 pathways $(52.63 \%)$ belonging to the "signal transduction" process and the remaining 9 pathways correspondingly categorized into "endocrine system", "cell growth and death", "cell communication", "metabolism system" and "immune system" processes. The close connection between module 1 and the other 5 modules indicates a potentially significant role of module 1 in maintaining normal biological function. Module 1 primarily reflected basic cellular signal transduction processes regulated by Sal B. Furthermore, the node representing the MAPK signaling pathway in module 1 showed the largest network connectivity with 32 other nodes in the pathway-pathway association network and always took part in cellular proliferation, differentiation and apoptosis biological processes (see Figure S1). Abnormal signaling pathways have been implicated in CVD [25]; the MAPK pathway has also been shown to play an important role in hy- pertrophy, cardiac remodeling after myocardial infarction, atherosclerosis and vascular restenosis [26]. Sal B may exert therapeutic effects by regulating the "signal transduction" pathway module.

In modules 2 and 5, "immune pathways" accounted for the largest proportion ( 0.75 and 0.43 , respectively). With the largest degree of network connectivity in module 5 , the "regulation of actin cytoskeleton" pathway had a significant role in several cellular processes such as migration, morphogenesis and endocytosis, which are involved in cellular immunity and inflammation [27]. Modules 2, 5 and 3 were dominated by "immune/inflammation" processes. Some studies had indicated that cardiovascular disease was caused mainly by atherosclerosis related to macrophages, $\mathrm{T}$ cells and other cells of the immune response, together with cholesterol infiltrating from the blood [28]. Although most pathways in module 3 belonged to the "immune system" process, some (such as the Toll-like receptor signaling pathway) also participated in cell apoptosis processes and were classified as "cell growth and death" pathways. The apoptosis pathway was the most connected node in module 3 , which may imply that this is the main function of the module. Apoptosis plays a key role in the pathogenesis of a variety of cardiovascular diseases to which the loss of terminally differentiated cardiac myocytes is a contributory factor. Cardiac myocytes undergoing apoptosis have been identified in tissue samples from patients suffering from myocardial infarction, diabetic cardiomyopathy, and endstage congestive heart failure. Evidence shows that inhibition of apoptosis was cardio-protective and could prevent the development of heart failure [29].

Module 6 mainly reflects ion transport processes. Among the nine pathways in module 6 , five $(55.6 \%)$ were associated with this function, of which the calcium-signaling pathway possessed the largest network connectivity. The vascular smooth muscle contraction pathway in module 6 regulated cellular $\mathrm{Ca}^{2+}$ concentration to stimulate vascular smooth muscle cell contraction, decreased vascular diameter and ultimately modulated blood flow and pressure. Abnormality within this pathway was potentially pathogenic for some forms of CVD.

Linking only with modules 1 and 2, module 4 had relatively little network connectivity and performed a specialist biological function. The vast majority $(92.59 \%)$ of pathways in this group were classified into the "metabolism" biological process that controls synthesis and metabolism of carbohydrates, lipids, proteins and nucleic acids. The glycolysis/gluconeogenesis pathway demonstrated the largest network connectivity in this module. In vivo, hormones and polysaccharides produced in the metabolism process mediated cell recognition, cell communication and signal transduction. Signal transduction pathways also regulated the normal metabolism process, so module 4 was closely connected with module 1 . From the characteristics of all six modules, it can be concluded that Sal B is involved in CVD 
via actions on cellular apoptosis, metabolism, immunity/ inflammation and ion transport.

Statistical analysis on the distribution of targets among the six modules showed that there were no enriched basic pathways in module 2, which contains fewer regulated proteins and predicted targets (Figure 3 ). Module 4 contained no regulated proteins. Among the six modules, module 5 contained the largest number of targets $(22 / 50$, or $\sim 44 \%)$, regulated proteins $(7 / 11$, or $\sim 63 \%)$, and the highest proportion of target-enriched basic pathways. To regulate CVD, Sal B tended to affect module 5 (representing "immunity/ inflammation") rather than modules 2 or 4 . In addition, a hyper-geometric test was taken to evaluate enrichment of the predicted targets and regulated proteins in each module. The outcomes demonstrated that "targets" and "regulated proteins" were highly enriched in module 5 with $P$ values of 0.00039 and 0.00027 , respectively. In module 4 , the $P$ value of "targets" was 0.04445 but there were no regulated proteins in the module so no statistical analysis was possible on this parameter. For all remaining modules, $P$ values were $>0.05$, suggesting that there was no significant enrichment of either targets or regulated proteins in these modules. Therefore, in the following section, we focus on module 5 ("immunity/ inflammation" biological process) to further study the regulatory function of Sal B.

\subsection{The regulation of Sal B on immune/inflammation physiological process}

In module 5, Sal B regulation of CVD is dominated by effects on "immunity/inflammation" physiological function processes, which account for the largest number of targets and regulated proteins. It has been demonstrated that an increase in the migration and proliferation of macrophages, $\mathrm{T}$ lymphocytes, and smooth muscle cells underlies some of

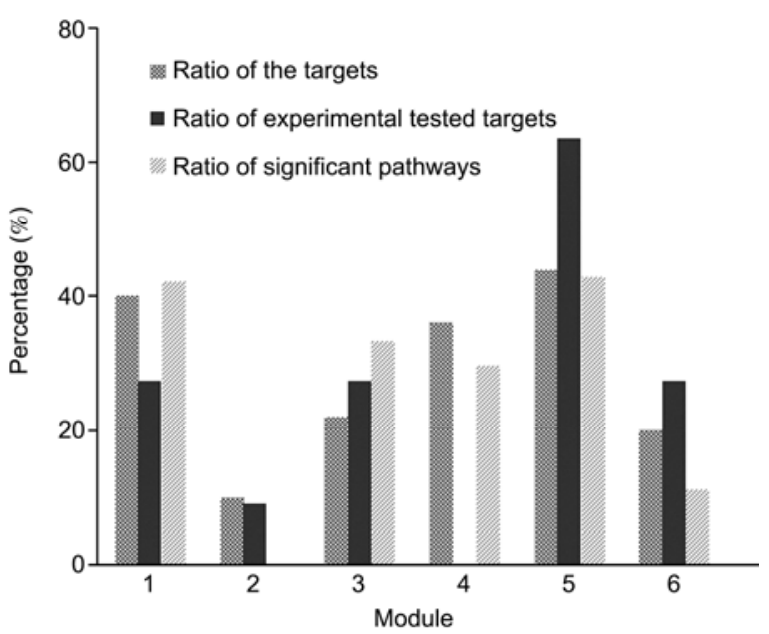

Figure 3 Statistical analysis of the number of targets, proven regulated proteins and target-enriched basic pathways in each module. $X$ axis represents the six modules and $Y$ axis represents the ratio of number of targets, regulated proteins and target-enriched pathways. the key cellular events in the atherogenic process, and this appears to be in response to the inflammatory activation of cells. This results in an increase in the secretion of cytokines, chemoattractants, and growth regulatory molecules [30]. Therefore, the efficacy of Sal B in treating CVD may lie in its regulation of the 14 basic pathways in the "immunity/ inflammation" physiological module. We constructed an "immunity/inflammation" physiological network based on 19 Sal B targets and 8 proven Sal B-regulated proteins in these 14 pathways and studied the therapeutic mechanism of Sal B in this network (Figure 4).

In Figure 4, Sal B is shown to regulate 27 proteins, thus modulating immune activation, inflammatory actions, cell adherence and thrombus occurrence, all of which contribute to CVD development. It shows that Sal B may regulate immune activation by influencing KLKB1, GF, RIK, IR, CD40, PI3K, AKT, GSK3 $\beta$, MEK1/2 and CaN proteins. Some evidence has suggested that Sal B decreases inflammation by inhibiting ICAM1, VCAM1 and NFkB [31]. In the coagulation cascade, Sal B possibly impacts AKT, PROC, REN and ACE to influence thrombus formation. Sal $B$ also decreases the fibrinolytic and anticoagulant potential of human umbilical vein endothelial cells by up-regulating the expression of t-PA (PLAT) and TM (THBD), which form complexes with F2, decreasing the amount of F2 and leading to thrombus formation [32]. In addition, Sal B maybe impact PLAUR, INSR, PGFR1 and $\beta$-Caterin to regulate cell adhesion.

\section{Conclusions}

We retrieved 58 predicted Sal B targets using a docking method, and 11 proven Sal B-regulated proteins were collected by manual curation in PubMed. From DrugBank, we defined 160 current FDA-approved CVD drugs and 193 corresponding targets. From the Sal B drug-target association network, we found that Sal B could impact ACE and REN in the renin-angiotensin-aldosterone system to relax blood vessels and regulate hypertension. Our pathway-pathway association network further demonstrated that Sal B can regulate immunity/inflammation, apoptosis, ion transport and basic metabolism physiological processes in the treatment of CVD. Of these, regulation of the immune/inflammation process may be the major molecular mechanism of Sal B.

Compared with protein-based analysis methods, we constructed a pathway-based network which was regulated by Sal B. This may increase our understanding of the regulatory mechanisms modulated by Sal B in the treatment of CVD by giving full consideration to all aspects of the relationship between pathways and proteins. This represents an alternative to traditional methods such as transcriptomics. Our method analyzed molecular mechanisms from enrichment modules, not just proteins. This should decrease the number 


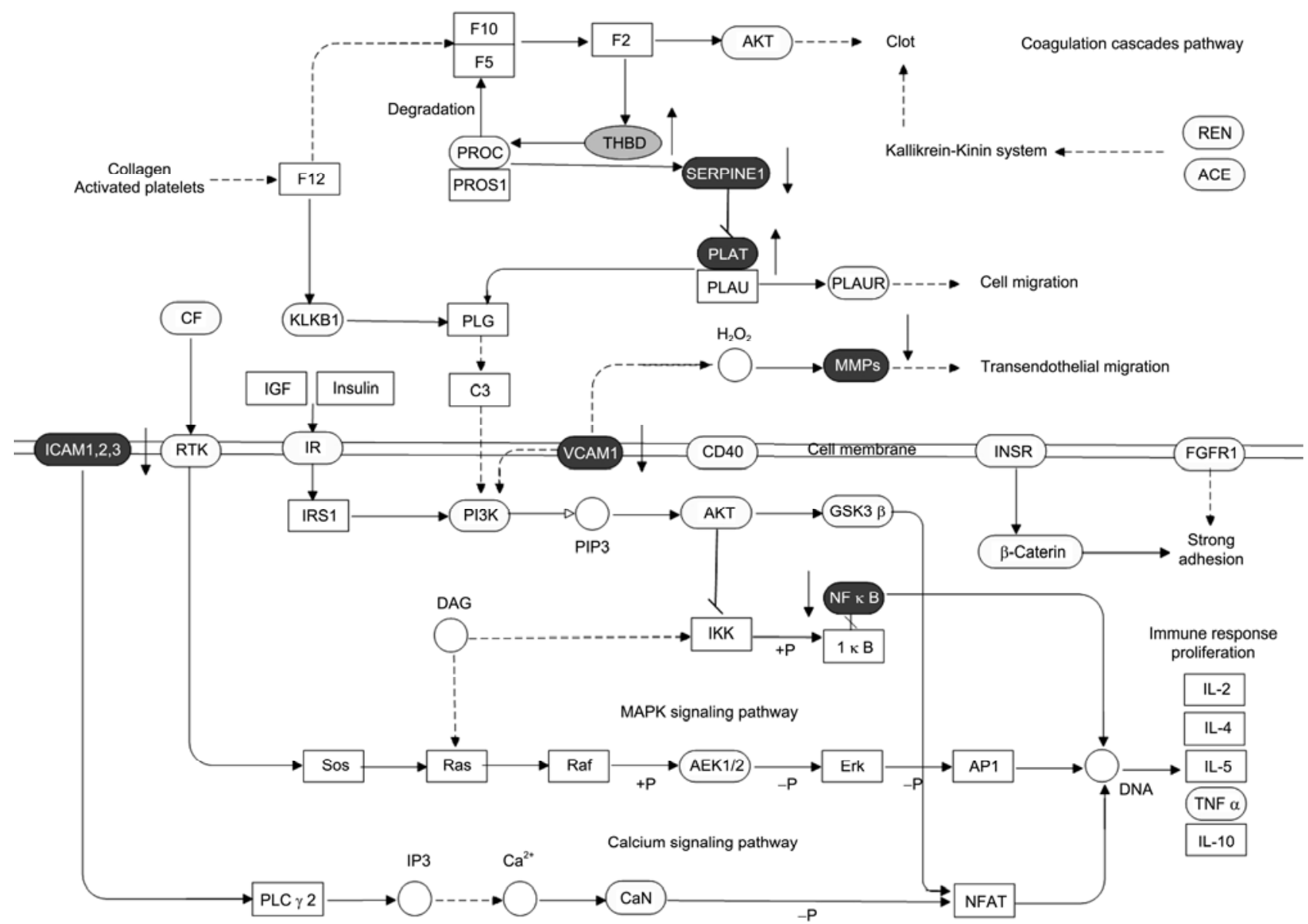

Figure 4 "Immunity/inflammation" physiological network regulated by Sal B. Grey rounded rectangle nodes represent computed Sal B targets; black rounded rectangle nodes represent proven SalB-regulated proteins. The grey ellipse node (THBD) was present in both sets of proteins.

of false positives and complement other methods. In summary, we hope that this new pathway-centric analysis method will advance our understanding of the mechanisms of action of bioactive herbal ingredients.

This work was supported by the National Natural Science Foundation of China (30900832), the Open Project Program Foundation of Key Laboratory of Liver and Kidney Diseases (Shanghai University of Traditional Chinese Medicine), the Program for New Century Excellent Talents in University (NCET-08-0399), the "Shu Guang" Project by Shanghai Municipal Education Commission and Shanghai Education Development Foundation (07SG22), and the National Science and Technology Major Project of China (2012ZX10005001).

1 Zhu M, Gao L, Li X, et al. The analysis of the drug-targets based on the topological properties in the human protein-protein interaction network. J Drug Targeting, 2009, 17: 524-532

2 Chen J Y, Yan Z, Shen C, et al. A systems biology approach to the study of cisplatin drug resistance in ovarian cancers. J Bioinform Comput Biol, 2007, 5: 383-405

3 Chu L, Chen B. Construction of a cancer-perturbed protein-protein interaction network for discovery of apoptosis drug targets. BMC Syst Biol, 2008, 2: 56

4 Yang L, Chen J, Shi L, et al. Identifying unexpected therapeutic targets via chemical-protein interactome. PLoS One, 2010, 5: e9568
5 Lamb J, Crawford E D, Peck D, et al. The connectivity map: Using gene-expression signatures to connect small molecules, genes, and disease. Science, 2006, 313: 1929-1935

6 Yue Q X, Cao Z W, Guan S H, et al. Proteomics characterization of the cytotoxicity mechanism of ganoderic acid D and computer- automated estimation of the possible drug target network. Mol Cell Proteomics, 2008, 7: 949-961

7 Barabási A, Oltvai Z N. Network biology: Understanding the cell's functional organization. Nat Rev Genet, 2004, 5: 101-113

8 Kanehisa M, Goto S, Furumichi M, et al. KEGG for representation and analysis of molecular networks involving diseases and drugs. Nucleic Acids Res, 2010, 38: D355-D360

9 Zhao J, Jiang P, Zhang W. Molecular networks for the study of TCM pharmacology. Brief Bioinform, 2010, 11: 417-430

10 Sucher $\mathrm{N} \mathrm{J}$. Insights from molecular investigations of traditional Chinese herbal stroke medicines: Implications for neuroprotective epilepsy therapy. Epilepsy Behav, 2006, 8: 350-362

11 Wang L, Zhou G, Liu P, et al. Dissection of mechanisms of Chinese medicinal formula Realgar-Indigo naturalis as an effective treatment for promyelocytic leukemia. Proc Natl Acad Sci USA, 2008, 105: 4826-4831

12 Lu Y, Liu X, Liang X, et al. Metabolomic strategy to study therapeutic and synergistic effects of tanshinone IIA, salvianolic acid B and ginsenoside $\mathrm{Rb} 1$ in myocardial ischemia rats. J Ethnopharmacol, 2011, 134: 45-49

13 Yang T, Lin F, Chen Y, et al. Salvianolic acid B inhibits low-density lipoprotein oxidation and neointimal hyperplasia in endothelium-denuded hypercholesterolaemic rabbits. J Sci Food Agric, 2011, 
91: $134-141$

14 Hartwell L H, Hopfield J J, Leibler S, et al. From molecular to modular cell biology. Nature, 1999, 402: C47-C52

15 Kuntz I D. Structure-based strategies for drug design and discovery. Science, 1992, 257: 1078-1082

16 Goodsell D S, Morris G M, Olson A J. Automated docking of flexible ligands: Applications of AutoDock. J Mol Recognit, 1996, 9: 1-5

17 Rarey M, Kramer B, Lengauer T, et al. A fast flexible docking method using an incremental construction algorithm. J Mol Biol, 1996, 261: 470-489

18 Chen Y Z, Zhi D G. Ligand-protein inverse docking and its potential use in the computer search of protein targets of a small molecule. Proteins, 2001, 43: 217-226

19 Ashburner M, Ball C A, Blake J A, et al. Gene ontology: Tool for the unification of biology. The gene ontology consortium. Nat Genet, 2000, 25: 25-29

20 Guimerà R, Nunes A L A. Functional cartography of complex metabolic networks. Nature, 2005, 433: 895-900

21 Newman M E J, Girvan M. Finding and evaluating community structure in networks. Phys Rev E Stat Nonlin Soft Matter Phys, 2004, 69: 026113

22 Zhang R, Xu X, Chen T, et al. An assay for angiotensin-converting enzyme using capillary zone electrophoresis. Anal Biochem, 2000, 280: 286-290

23 Gao X, Xu D, Deng Y, et al. Screening of angiotensin converting enzyme inhibitors from Salvia miltiorrhizae (in Chinese). China Journal of Chinese Materia Medica, 2004, 29: 359-362

24 Cline M S, Smoot M, Cerami E, et al. Integration of biological networks and gene expression data using Cytoscape. Nat Protoc, 2007, 2: 2366-2382

25 Hunter J J, Chien K R. Signaling pathways for cardiac hypertrophy and failure. N Engl J Med, 1999, 341: 1276-1283

26 Muslin A J. MAPK signalling in cardiovascular health and disease: Molecular mechanisms and therapeutic targets. Clin Sci, 2008, 115: 203-218

27 Saarikangas J, Zhao H, Lappalainen P. Regulation of the actin cytoskeleton-plasma membrane interplay by phosphoinositides. Phys Rev, 2010, 90: 259-289

28 Hansson G K, Hermansson A. The immune system in atherosclerosis. Nat Immunol, 2011, 12: 204-212

29 Lee Y, Gustafsson A B. Role of apoptosis in cardiovascular disease. Apoptosis, 2009, 14: 536-548

30 Rosenfeld M E. Cellular mechanisms in the development of atherosclerosis. Diabetes Res Clin Pract, 1996, 30: 1-11

31 Chen Y H, Lin S J, Ku H H, et al. Salvianolic acid B attenuates VCAM-1 and ICAM-1 expression in TNF-alpha-treated human aortic endothelial cells. J Cell Biochem, 2001, 82: 512-521

32 Shi C, Huang $\mathrm{H}$, Wu H, et al. Salvianolic acid B modulates hemostasis properties of human umbilical vein endothelial cells. J Cell Biochem, 2007, 119: 769-775

Open Access This article is distributed under the terms of the Creative Commons Attribution License which permits any use, distribution, and reproduction in any medium, provided the original author(s) and source are credited.

\section{Supporting Information}

Figure S1 Pathway-pathway association network of Sal b.

Table S1 Pathway information of Sal b

The supporting information is available online at csb.scichina.com and www.springerlink.com. The supporting materials are published as submitted, without typesetting or editing. The responsibility for scientific accuracy and content remains entirely with the authors. 